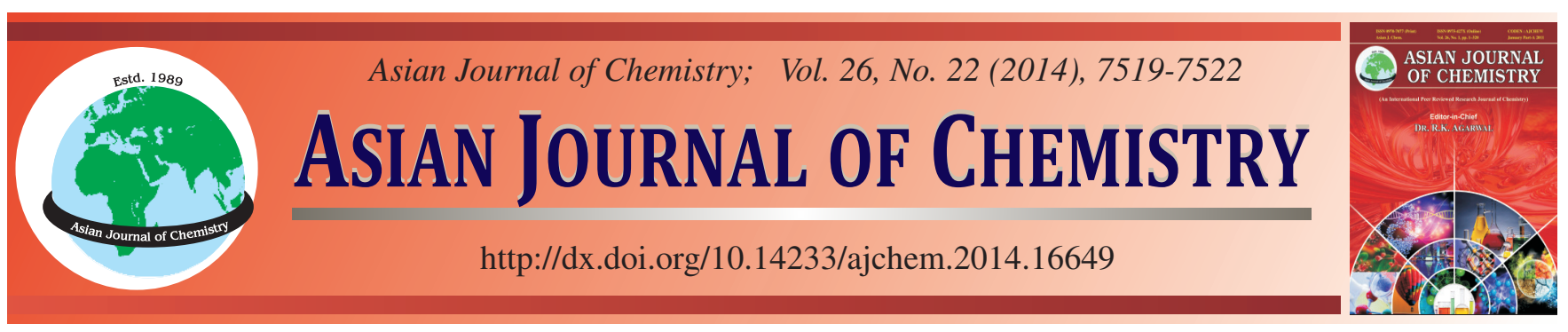

\title{
Synthesis of Polyaniline Composites with Grinded Leaves of Polyalthia longifolia, Syzygium cumini, Alstonia scholaris and Madhuca longifolia and Study of Their Structural, Electrical and Dielectric Properties
}

\author{
Farah Kanwal ${ }^{1, *}$, Aisha Batool ${ }^{2}$, Sadia Rasool $^{1}$, Shahzad Naseem $^{2}$ and Rabia Rehman ${ }^{1}$
}

${ }^{1}$ Institute of Chemistry, University of the Punjab, Lahore, Pakistan

${ }^{2}$ Centre of Excellence in Solid State Physics, University of the Punjab, Lahore, Pakistan

*Corresponding author: Fax: +92 42 99230998; E-mail: farahkchem@yahoo.com

Polyaniline composites has successfully been synthesized with well grinded leaves of Polyalthia longifolia, Syzygium cumini, Madhuca longifolia and Alstonia scholaris in an acidic medium by chemical oxidation method at room temperature. Fourier transform infrared (FT-IR) spectroscopy confirmed the formation of composites. All prepared samples were characterized for their electrical and dielectric properties. Polyaniline/Polyalthia longifolia composite offer enhanced dielectric properties with dielectric constant four times greater than pure polyaniline, suitable for its application as passivation layer for thin film transistor.

Keywords: Polyaniline, Grinded leaves powder, Composites, AC conductivity, Dielectric constant.

\section{INTRODUCTION}

Tremendous research has been carried out on conjugated polymers since 1977 after the unexpected discovery that all polymers are not insulators ${ }^{1}$. Charge transportation in that class of polymers is carried out through doping to conduct charge by chain deformation and relaxation phenomena; followed by addition of electrons or holes to produce variety of localized defects such as solitons, polarons or bipolarons ${ }^{2}$.

Among inherent conducting polymers such as polypyrrole, polyacetylene, polythiophene and their derivatives, polyaniline (PANI) has received passionate attention since past two decades due to its environmental stability, non-toxic behaviour, inexpensive, easy to synthesis and good conductivity. Polyaniline can be prepared chemically or electrochemically in an acidic medium by using some appropriate oxidant. Polyaniline exists in two forms named as emeraldine base (EB) and leucoemeraldine base (LB) in which emeraldine salt is its most conductive form to offer numerous technological applications as sensors, electromagnetic interference shielding ${ }^{3-6}$, microwave absorbent and secondary batteries ${ }^{7}$. However, charge-carrier mobility of native inherent conducting polymers is rather low with poor processibility and fusibility for their direct use in electronic devices. Research efforts are performed to overcome these drawbacks. Among them preparing blends and composites is an extensive strategy to approach desired properties $^{8,9}$. Prodigious research papers have been published to elaborate the formation of their composites via different synthetic routes with insulating polymers, transition metal oxides, metals or magnetic nanoparticles and their respective applications ${ }^{10-16}$. Among several polymer matrix fillers, plants grinded leaves can be an attractive selection to modify properties of polyaniline.

To the best of our knowledge, nothing has been reported the structural, electrical and dielectric properties of polyaniline composites with grinded leaves of Polyalthia longifolia (PL), Syzygium cumini (SC), Madhuca longifolia (ML) and Alstonia scholaris (AL). In this project, polyaniline is used as polymer matrixand the grinded leaves act as fillerby chemical oxidation method to study their effect on structural, electrical and dielectric properties of polyaniline.

\section{EXPERIMENTAL}

Aniline (Riedel-deHaën) was distilled twice at atmospheric pressure just before use. Iron chloride $\left(\mathrm{FeCl}_{3}\right)(\mathrm{AR}$ grade) was used as received to polymerize aniline monomer. All other chemicals were of analytical grade. Double distilled water was used for all dilutions. The leaves of Polyalthia longifolia, Syzygium cumini, Madhuca longifolia and Alstonia scholaris were collected from home institute.

Synthesis of polyaniline: Polyaniline was synthesized by chemical oxidation of aniline using ferric chloride as an oxidizing agent in an acidic medium. 0.12 mole of aniline was dissolved in $375 \mathrm{~mL}$ of $1 \mathrm{~N} \mathrm{HCl}$ acid to form acidic aniline. 
$0.3 \mathrm{~mol}$ of ferric chloride solution dissolved in $100 \mathrm{~mL}$ of distilled water was then added drop wise to the above acidic solution and allowed to stir for $24 \mathrm{~h}$. Dark green precipitates were formed, filtered by using pyrex sintered glass crucible and vacuum apparatus and washed several times with distilled water, methanol and acetone in order to removeunreacted salts and oligomers. Further these precipitates were dried in vacuum oven for $48 \mathrm{~h}$. The calculated yield of the polyaniline was approximately $60 \%$.

Preparation of polyaniline composites with grinded leaves of Polyalthia longifolia, Syzygium cumini, Alstonia scholaris and Madhuca longifolia: Initially the leaves of Polyalthia longifolia, Syzygium cumini, Madhuca longifolia and Alstonia scholaris were washed thoroughly with excess amount of tap water and then dried in vacuum oven at $50{ }^{\circ} \mathrm{C}$ for $12 \mathrm{~h}$. Then these were separately grinded to transform them into powder phase to synthesize composites.

The composite of polyaniline with Polyalthia longifolia was synthesized by dispersing $20 \mathrm{wt}$. \% of Polyalthia longifolia of polyaniline yield in the polymerization solution and stirred further for $24 \mathrm{~h}$ under inert atmosphere and proceed the polymerization solution as described above. The obtained product was named as polyaniline/Polyalthia longifolia composite. Other composites of polyaniline with Syzygium cumini, Alstonia scholaris and Madhuca longifolia were synthesized by following the same above procedureto get polyaniline/ Syzygium cumini, polyaniline/Alstonia scholaris and polyaniline/Madhuca longifolia composites; exhibiting 86, 78 and 82 yield \% ages respectively. Finally all these composites were well grinded in agate pastel mortal and then pelletized of diameter $16 \mathrm{~mm}$ by applying pressure of 7 tones using Apex hydraulic press and then subjected them to desired characterizations.

\section{RESULTS AND DISCUSSION}

The FT-IR spectra of these composites were recorded by FT-IR (Perkin Elmer RXI) operated at room temperature in the normal IR region 4000-650 and $4 \mathrm{~cm}^{-1}$ band resolutions ${ }^{17}$. The current-voltage(I-V) characteristics were calculated by standard four probe method using semiconductor characterization system (Keithley 4200-SCS). Dielectric measurements were carried out in the frequency range of 102-106 Hz using the Wayne Kerr dielectric analyzer 6500-B model at $300 \mathrm{~K}$.

FT-IR analysis: The FT-IR spectra of polyaniline and its composites are shown in Fig. 1. The characteristic peaks at 1625 and $1483 \mathrm{~cm}^{-1}$ correspond to stretching vibration of quinoid ring and benzenoid ring of polyaniline in emeraldine base form respectively ${ }^{18}$. Other absorption bands of polyaniline are at $1292 \mathrm{~cm}^{-1}$ (C-N stretching of the secondary amine), $1047 \mathrm{~cm}^{-1}\left(\mathrm{Q}=\mathrm{N}=\mathrm{Q}\right.$ stretching band) and $895 \mathrm{~cm}^{-1}$ (out-of-plane stretching vibration of $\mathrm{C}-\mathrm{H})^{19,20}$. After composites formation, all the characteristic peaks of polyaniline has shifted towards lower frequencies indicating some physiochemical interactions between polyaniline back bone and dispersant particles to enhance its properties ${ }^{21}$.

Electrical conductivity: The electrical DC conductivity was measured by standard four points probe method to avoid the errors caused from contact resistance. Resistivity of poly-

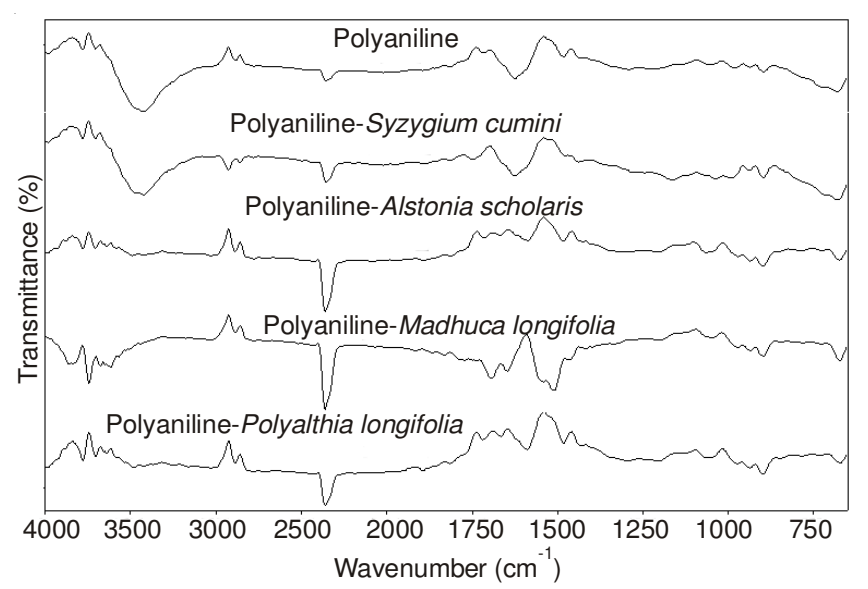

Fig. 1. FT-IR spectra of pure polyaniline and its composites

aniline decreases by forming its composites with Polyalthia longifolia, Syzygium cumini, Madhuca longifolia and Alstonia scholaris. I-V plot of polyaniline/Polyalthia longifolia showed good linearity over wide range of applied voltage (Fig. 2) and offered maximum value of conductivity $\left(4.01 \times 10^{-3} \mathrm{~S} \mathrm{~cm}^{-1}\right)$, $1000 \mathrm{~S} \mathrm{~cm}^{-1}$ greater than neat polyaniline (Fig. 3) (Table-1). The conductivity values of other composites listed in Table-1 illustrate the effect of different dispersions on electrical properties of polyaniline. The conductivity of all the composites is higher than that of pure polyaniline except polyaniline/ Alstonia scholaris composite. Lowest conductivity of polyaniline/Alstonia scholaris composite may be attributed to poor connectivity between the polymer and grains to decrease its compactness. The polymer chains are not supported by the grinded leaves particles that reduce its packing density and conductivity in parallel ${ }^{22}$.

\begin{tabular}{lc}
\multicolumn{2}{c}{ TABLE-1 } \\
VARIATION IN ELECTRICAL PROPERTIES OF \\
POLYANILINE WITH DIFFERENT DISPERSANTS \\
\hline \multicolumn{1}{c}{ Entities } & DC conductivity \\
& $\left(\mathrm{S} \mathrm{cm}^{-1}\right)$ \\
\hline Polyaniline & $8.07 \times 10^{-4}$ \\
Polyaniline/Polyalthia longifolia & $4.01 \times 10^{-3}$ \\
Polyaniline/Syzygium cumini & $3.14 \times 10^{-4}$ \\
Polyaniline/Alstonia scholaris & $8.97 \times 10^{-5}$ \\
\hline
\end{tabular}

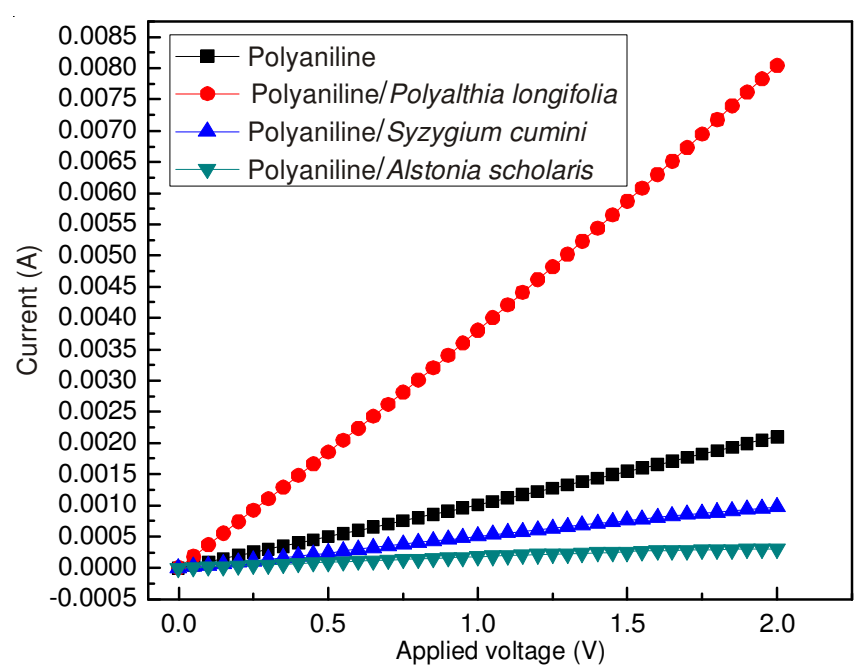

Fig. 2. Typical I-V plots shown by polyaniline and its composites 


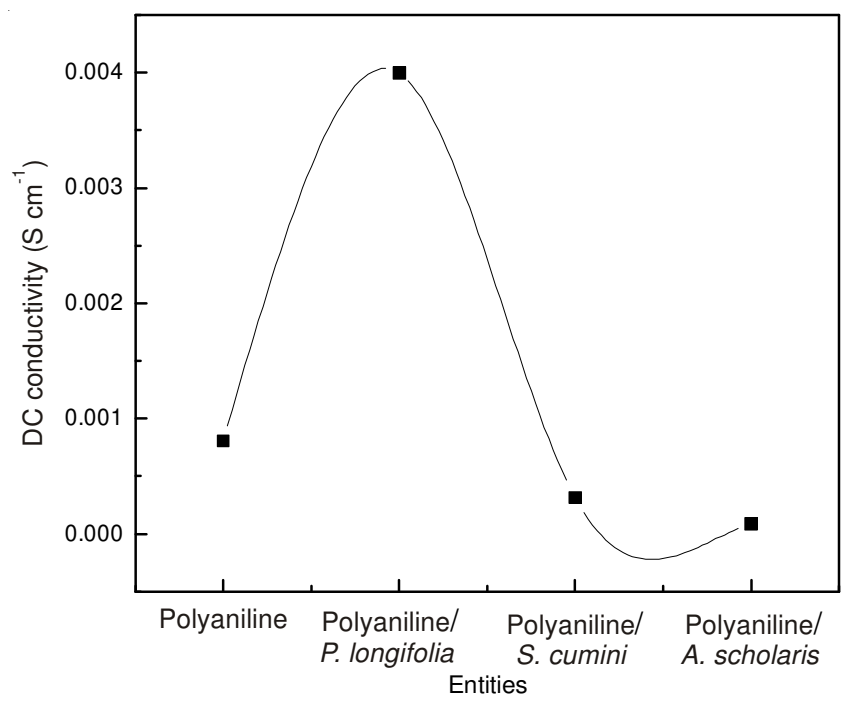

Fig. 3. Variation in DC conductivity of polyaniline and its composites with grinded leaves

Dielectric measurements: The dielectric properties of all the samples are measured in the frequency range of $1 \mathrm{kHz}$ to $10 \mathrm{MHz}$ at room temperature. It is seen that dielectric constant $\left(\varepsilon^{\prime}\right)$ of all composites decrease sharply with the increase of frequency and becomes independent at higher applied frequencies. Fig. 4 shows that polyaniline/Polyalthia longifolia possessed highest $\varepsilon^{\prime}$ than other three composites which is about 4 times greater than that of pristine polyaniline. The maximum value of $\varepsilon^{\prime}$ attained is 68 for the former and 37, 26 and 22 for polyaniline/Madhuca longifolia, polyaniline/Polyalthia longifolia and polyaniline/Alstonia scholaris, respectively (Table-2). At low test frequencies, electrons hoped easily by following the applied field that result in large polarization and consequently the large dielectric constant values ${ }^{23}$. But at high test frequencies the dipole moment could not follow the applied frequency that consequently causes to drop in their dielectric constants. It is also evident from Fig. 5 that frequency-dependent $\varepsilon^{\prime}$ behaviour of polyaniline/Alstonia scholaris composite is some what changed then others. Its dielectric constant is found to be stable in terms of frequencies from 1 to $7.5 \mathrm{KHz}$ and then decreases steeply. Such behaviour is consistent with the $\varepsilon^{\prime}$ behavior of polymer-ceramic composites and cermet percolation composites ${ }^{24}$.

Dependence of tangent of dielectric loss angle $(\tan \delta)$ and dielectric loss factor $\left(\varepsilon^{\prime \prime}\right)$ as a function of applied frequency at room temperature are plotted in Figs. 6 and 7, respectively. At lower frequencies the dipoles respond swiftly to monitor the field and dipole polarization has its maximum value, so offered highest $\tan \delta$ and $\varepsilon^{\prime \prime}$. Dielectric values achieved minimum at higher frequencies as the field could not induce the dipole

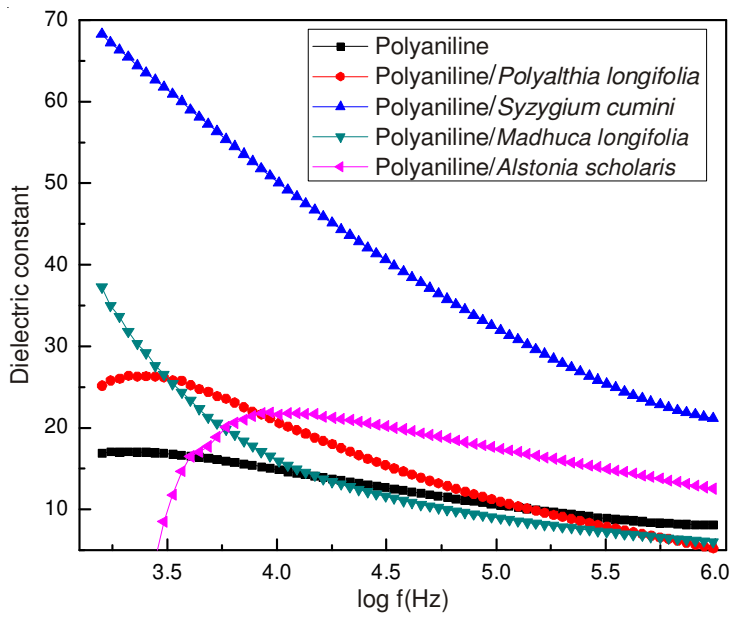

Fig. 4. Frequency-dependent room temperature dielectric constants of polyaniline composites

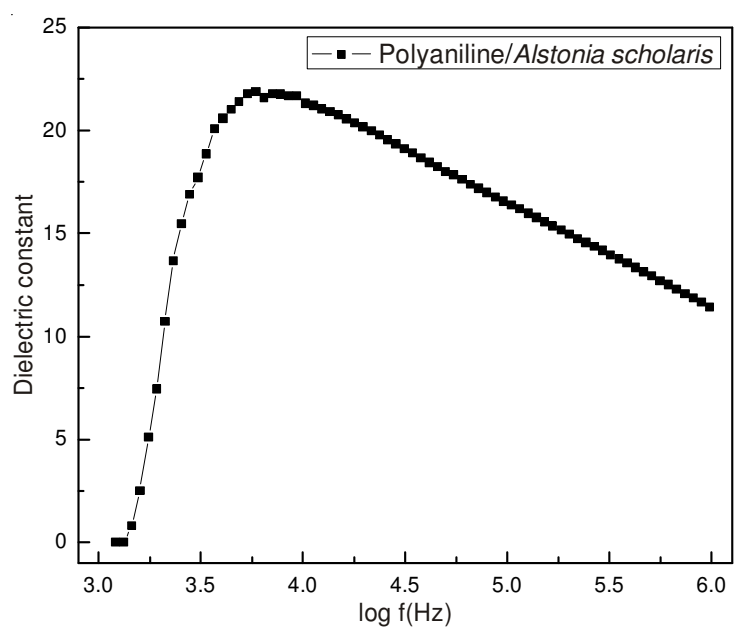

Fig. 5 Frequency-dependent room temperature dielectric constant of polyaniline/alstonia scholaris composite

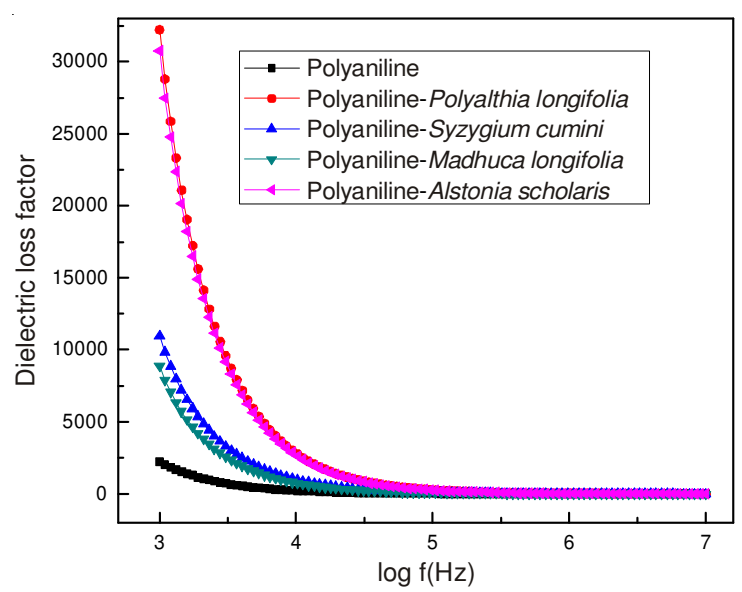

Fig. 6. Frequency-dependent room temperature dielectric loss factor of polyaniline composites

TABLE-2

ROOM TEMPERATURE DIELECTRIC STUDIES OF POLYANILINE COMPOSITES AT TWO TEST FREQUENCIES

\begin{tabular}{|c|c|c|c|c|c|c|}
\hline \multirow{2}{*}{ Entity } & \multicolumn{3}{|c|}{$1 \mathrm{KHz}$} & \multicolumn{3}{|c|}{$500 \mathrm{KHz}$} \\
\hline & $\varepsilon^{\prime}$ & $\tan \delta$ & $\varepsilon^{\prime \prime}$ & $\varepsilon^{\prime}$ & $\tan \varepsilon$ & $\varepsilon^{\prime \prime}$ \\
\hline Polyaniline & 16.39 & 68.63 & 0.0021 & 8.40 & 0.78 & 6.58 \\
\hline Polyaniline-Polyalthia longifolia & 23.68 & 600.90 & 0.0003 & 6.78 & 8.13 & 5.70 \\
\hline Polyaniline-Syzygium cumini & 82.65 & 80.53 & 0.0001 & 23.18 & 1.15 & 26.80 \\
\hline Polyaniline-Madhuca longifolia & 37.12 & 125.32 & 0.0009 & 6.67 & 2.35 & 15.70 \\
\hline Polyaniline-Alstonia scholaris & 2.86 & 1993 & 0.0002 & 12.93 & 4.04 & 52.20 \\
\hline
\end{tabular}




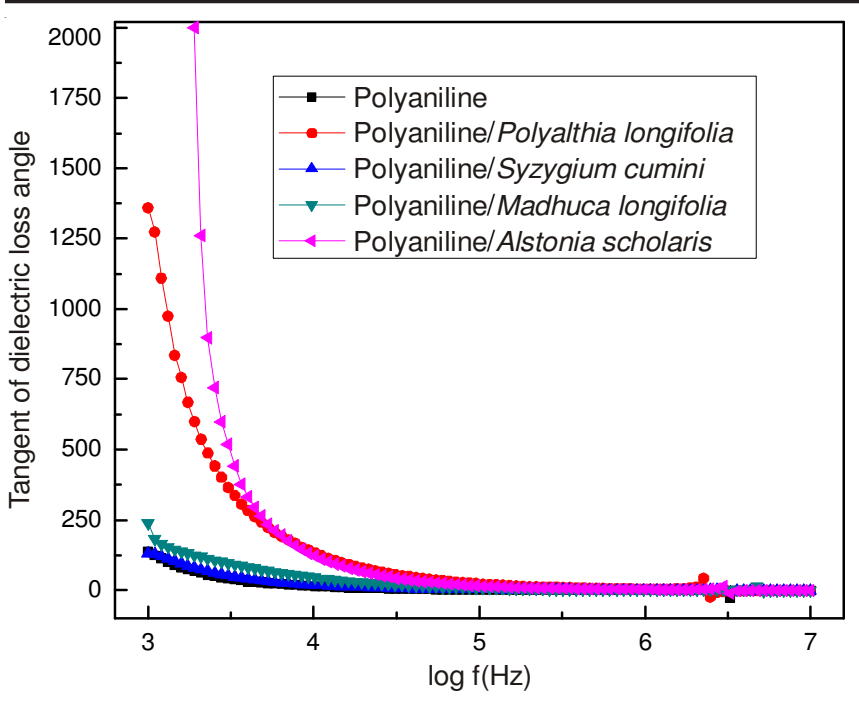

Fig. 7. Frequency-dependent room temperature tangent of dielectric loss angle of polyaniline composites

moment and the dipoles were unable to bring into line themselves in the direction of applied field due to inertia so dipole polarizability found to be minimum.

\section{Conclusion}

Polyaniline and its composites with grinded leaves of Polyalthia longifolia, Syzygium cumini, Madhuca longifolia and Alstonia scholaris was prepared via chemical oxidation. FT-IR analysis confirmed the formation of polyaniline and its composites by characteristics polyaniline absorption bands and corresponding shift of peaks towards lower frequencies to indicate some physiochemical interactions developed between polyaniline and grinded leaves grains. Polyalthia longifolia provided homogeneous dispersion to support polymer chains and to form conductive path ways to enhance its conductivity to maximum. However polyaniline/Polyalthia longifolia composite possessed highest dielectric constant value of 68 that is four times greater than pristine polyaniline and can be used as low dielectric constant passivation layer in thin film transistors.

\section{REFERENCES}

1. J. Huang, Pure Appl. Chem., 78, 15 (2006).

2. J. Aguilar-Hernández and K. Potje-Kamloth, J. Phys. D Appl. Phys., 34, 1700 (2001).

3. Q. Tang, X. Sun, Q. Li, J. Lin and J. Wu, J. Mater. Sci., 44, 849 (2009).

4. J. Jiang, L. Ai and L. Li, J. Phys. Chem. B, 113, 13 (2009).

5. R. Khan, P. Khare, B.P. Baruah, A.K. Hazarika and N.C. Dey, Adv. Chem. Eng. Sci., 1, 37 (2011).

6. S.A. Ghani and H.C. Young, J. Physiol. Sci., 21, 81 (2010).

7. S. Sen, A. Gok and H. Gulce, J. Appl. Polym. Sci., 106, 3852 (2007).

8. D. Muller, J.S. Mandelli, J.A. Marins, B.G. Soares, L.M. Porto, C.R. Rambo and G.M.O. Barra, Cellulose, 19, 1645 (2012).

9. M. G-Balcázar, R. Ortega, F. Castaneda, L.G. Arriaga and J.L. Garcia, Int. J. Electrochem. Soc., 6, 4667 (2011).

10. C.S.S. Sangeeth, P. Jiménez, A.M. Benito, W.K. Maser and R. Menon, J. Appl. Phys., 107, 103719 (2010).

11. T.B. Phan, T.T. Pham and T.T.T. Mai, Adv. Nat. Sci.: Nanosci. Nanotechnol., 4, 015015 (2013).

12. X.-X. Xu, Z.-P. Cui, J. Qi and X.-X. Liu, J. Chem. Soc., Dalton Trans., 42, 4031 (2013).

13. Y.-N. Koh, K.P. Sambasevam, R. Yahya and S.-W. Phang, Polym. Composites, 34, 1186 (2013).

14. M. Rahaman, T.K. Chaki and D. Khastgir, Polym. Eng. Sci., 54, 1632 (2014).

15. S.H. Shumaila, S. Parveen, J. Ali, A. Kumar and M. Husain, Polym. Composites, 34, 1298 (2013).

16. X.J. Yang, J.Y. Li and Y.P. Lei, Adv. Mater. Res., 668, 17 (2013).

17. P.S. Kalsi, Spectroscopy of Organic Compounds, Wiley Eastern Limited, New Delhi, edn 6, pp. 66-68 (2006)

18. T.-H. Hsieh, K.-S. Ho, C.-H. Huang, Y.-Z. Wang and Z.-L. Chen, Synth. Metals, 156, 1355 (2006).

19. D. Zhang, Polym. Test., 26, 9 (2007).

20. G. Zhang, X. Li, H. Jia, X. Pang, H. Yang, Y. Wang and K. Ding, Int. J. Electrochem. Sci., 7, 819 (2012).

21. A. Batool, F. Kanwal, M. Imran, T. Jamil and S.A. Siddiqi, Synth. Met., 161, 2753 (2012).

22. R. Gangopadhyay and A. De, Eur. Polym. J., 35, 1985 (1999).

23. T.K. Vishnuvardhan, V.R. Kulkarni, C. Basavaraja and S.C. Raghavendra, Bull. Mater. Sci., 29, 77 (2006).

24. J. Xu, K.-S. Moon, C. Tison and C.P. Wong, IEEE Trans. Adv. Packag., 29, 295 (2006). 